\title{
BALL COVERING ON MANIFOLDS WITH NONNEGATIVE RICCI CURVATURE NEAR INFINITY
}

\author{
ZHONG-DONG LIU
}

(Communicated by Jonathan M. Rosenberg)

\begin{abstract}
Let $M$ be a complete open Riemannian manifold with nonnegative Ricci curvature outside a compact set $B$. We show that the following ball covering property (see [LT]) is true provided that the sectional curvature has a lower bound:

For a fixed $p_{0} \in M$, there exist $N>0$ and $r_{0}>0$ such that for $r \geq r_{0}$, there exist $p_{1}, \ldots, p_{k} \in \partial B_{p_{0}}(2 r), k \leq N$, with

$$
\bigcup_{j=1}^{k} B_{p_{j}}(r) \supset \partial B_{p_{0}}(2 r) .
$$

Furthermore $N$ and $r_{0}$ depend only on the dimension, the lower bound on the sectional curvature, and the radius of the ball at $p_{0}$ that contains $B$.
\end{abstract}

\section{INTRODUCTION}

On a complete open Riemannian manifold with nonnegative Ricci curvature, the volume comparison of Bishop and Gromov [BC, GLP] is a very powerful tool. It can be used to show that the geometric growth of its ends is well controlled; namely,

(a) The volume of the geodesic ball $B_{p}(r)$ is bounded above by $C \cdot r^{n}$, where $n$ is the dimension of the manifold and $C$ is the volume of the unit ball in $R^{n}$.

(b) The volume of $B_{p}(r)$ grows at least linearly.

(c) There are a bounded number of ends (Cheeger-Gromoll's splitting theorem implies that there are at most two ends).

(d) Every end has at most linear diameter growth ([AG]).

(e) There is a ball covering property: for a fixed point $p$ and any large $r$, there are a bounded number of points $p_{1}, \ldots, p_{k} \in \partial B_{p}(2 r)$, where the number $k$ is independent of $r$, such that

$$
\partial B_{p}(2 r) \subset \bigcup_{j=1}^{k} B_{p_{j}}(r) .
$$

Received by the editors May 29, 1990 and, in revised form, August 22, 1990. 1980 Mathematics Subject Classification (1985 Revision). Primary 53C20.

Key words and phrases. Ricci curvature, end, volume comparison, geodesic ball. 
When the global condition $\mathrm{Ric}_{M} \geq 0$ is relaxed to $\mathrm{Ric}_{M-B} \geq 0$, where $B$ is a compact set, the relative volume comparison is weakened. However, it is still fairly easy to show that (a) and (b) remain true, even though the constant $C$ now depends on the manifold. Moreover, the ball covering property (e), if true, would imply (c) and (d).

In their paper [LT], P. Li and L. Tam show that (e) is true if the sectional curvature is nonnegative outside a compact set. This ball covering property plays a crucial role in their study of positive harmonic functions on their class of manifolds. In that paper they ask whether (e) remains true if sectional curvature is replaced by Ricci curvature.

Here, we will prove that if in addition to assuming $\operatorname{Ric}_{M-B} \geq 0$, one assumes that the sectional curvature is bounded below, then (e) is true. Furthermore, the bound can be estimated in terms of the dimension, the lower bound on the sectional curvature, and the radius of the ball $B_{p}(D)$ that contains the compact set $B$.

Theorem. Let $M^{n}$ be a complete Riemannian manifold with nonnegative Ricci curvature outside a compact set $B$. Assume also that the sectional curvature has a lower bound $H>-\infty$. If $B \subset B_{p_{0}}(D)$, then there exist $N>0$ and $r_{0}>0$ depending only on $n$ and $H \cdot D^{2}$ such that for $r \geq r_{0}$, we can find $p_{1}, \ldots, p_{k} \in \partial B_{p_{0}}(2 r), k \leq N$, with

$$
\bigcup_{j=1}^{k} B_{p_{j}}(r) \supset \partial B_{p_{0}}(2 r) \text {. }
$$

\section{Preliminaries}

Volume comparison. Let $M$ be an $n$-dimensional Riemannian manifold. Let $B_{p}(r)$ denote the geodesic ball of radius $r$ at $p \in M$. Put $A_{p}(R, r):=B_{p}(R)-$ $\overline{B_{p}(r)}, R>r>0 . \quad V_{p}(r):=\operatorname{vol}\left(B_{p}(r)\right), V_{p}(R, r):=\operatorname{vol}\left(A_{p}(R, r)\right)$. We use $V^{H}(r)$ to denote the volume of a ball of radius $r$ in the space form of constant curvature $H$ of the same dimension. $V^{H}(R, r):=V^{H}(R)-V^{H}(r)$. Then we have the well-known relative volume comparisons (see [GLP]).

Lemma 1. If on $B_{p}(R)$, Ric $\geq(n-1) H$ and $0<r<R$, then

$$
\begin{gathered}
V_{p}(R) / V_{p}(r) \leq V^{H}(R) / V^{H}(r), \\
V_{p}(R, r) / V_{p}(R) \leq V^{H}(R, r) / V^{H}(R),
\end{gathered}
$$

and

$$
V_{p}(R, r) / V_{p}(r) \leq V^{H}(R, r) / V^{H}(r) .
$$

There is also a relative volume comparison on star-shaped sets. A star-shaped set $S_{p}$ at $p$ is a set containing $p$ such that whenever $x \in S_{p}$ is not on the cut-locus of $p$, any point on the minimal geodesic joining $p$ and $x$ is also in $S_{p}$. We then have [CGT, $\S 4$, Remark 4.1].

Lemma 2. If $S_{p}$ is a star-shaped set and $\operatorname{Ric} \mid S_{p} \geq(n-1) H$, then for $R>r>0$ we have

$$
\operatorname{Vol}\left(S_{p} \bigcap B_{p}(R)\right) / \operatorname{Vol}\left(S_{p} \bigcap B_{p}(r)\right) \leq V^{H}(R) / V^{H}(r) .
$$

Excess functions. The excess was first introduced in [AG]. 
Let $\left(\gamma_{1}, \gamma_{2}, \gamma_{3}\right)$, where each $\gamma_{i}$ is minimal, form a geodesic triangle in $M$. $\gamma_{1}(0)=\gamma_{2}(0)=p, \alpha=\angle\left(\gamma_{1}^{\prime}(0), \gamma_{2}^{\prime}(0)\right)$ and $l_{i}:=$ length of $\gamma_{i}$. The excess function is defined to be $e:=l_{1}+l_{2}-l_{3}$. Let $h$ denote the altitude of the triangle with respect to the side $\gamma_{3}$, i.e., $h:=d\left(p, \gamma_{3}\right)$. The triangle inequality implies that $h \geq e / 2$.

Lemma 3. Let $M$ be the two-dimensional space form of constant curvature -1 . Suppose in the above geodesic triangle, $l_{1}=l_{2}=a, l_{3}=c$. Then the excess function $e$ has a limit, as $a \rightarrow \infty$, if $\alpha>0$ :

$$
\lim _{a \rightarrow \infty} e=2 \ln (\csc (\alpha / 2)) \text {. }
$$

When $\alpha=\pi, e \equiv 0$ and when $\alpha=0, e \equiv 2 a$.

Proof. If we draw the minimal geodesic $\tau$ from $p$ to the middle of $\gamma_{3}$, then the triangle is divided into two identical right triangles. We apply the law of sines in hyperbolic geometry to each right triangle:

$$
\frac{\sinh (a)}{\sinh (c / 2)}=\frac{\sin (\pi / 2)}{\sin (\alpha / 2)}
$$

Note that as $a \rightarrow \infty$, so does $c$. Taking limits in the above equation yields:

$$
\lim _{a \rightarrow \infty}(a-c / 2)=\ln (\csc (\alpha / 2)) \text {. }
$$

Thus

$$
\lim _{a \rightarrow \infty} e=\lim _{a \rightarrow \infty}(2 a-c)=2 \ln (\csc (\alpha / 2)) .
$$

This completes the proof of Lemma 3.

Remark 1. We observe the following properties about the excess function $e$ that will be useful later.

(i) By the triangle inequality, if $\alpha$ is held fixed while the lengths of $\gamma_{1}$ and $\gamma_{2}$ are increased, then the excess $e$, will increase as well.

Let $M_{-1}$ be the two-dimensional space form of constant curvature -1 and $M$ a complete manifold with curvature bounded below by -1 .

(ii) Construct a family of geodesic triangles $\left(\bar{\gamma}_{1}, \bar{\gamma}_{2}, \bar{\gamma}_{3}\right)$ in $M_{-1}$ as described in Lemma 3 , with $\bar{l}_{1}, \bar{l}_{2}$ fixed and $\bar{\alpha}$ variable. Then as $\bar{\alpha}$ decreases, $\bar{e}$ increases.

(iii) If $\left(\gamma_{1}, \gamma_{2}, \gamma_{3}\right)$ and $\left(\bar{\gamma}_{1}, \bar{\gamma}_{2}, \bar{\gamma}_{3}\right)$ are geodesic triangles in $M$ and $M_{-1}$ respectively with

$$
\text { length }\left(\gamma_{i}\right)=\operatorname{length}\left(\bar{\gamma}_{i}\right), i=1,2 \text {, and } \alpha=\bar{\alpha},
$$

then by Toponogov's Theorem, $e \geq \bar{e}$.

\section{PROOF OF THE THEOREM}

In this section we prove the theorem stated in $\S 1$.

We assume that all geodesics are parametrized by arclength.

Proof of the theorem. If $H \geq 0$, i.e., $K_{M} \geq 0$, the theorem is already known. So we assume that $H<0$. If we multiply the metric on $M$ by $\sqrt{-H}$, then for the new metric, $K \geq-1$, and $B \subset B_{p_{0}}(\sqrt{-H} \cdot D)$. This normalization does not affect the validity of the ball covering property. Thus we put $D_{1}=\sqrt{-H} \cdot D$ and work with the rescaled metric. 
Take an $\alpha>0$ small enough so that in Lemma 3 we have $\lim _{a \rightarrow \infty} e \geq 4 D_{1}$. Then there exists $\alpha_{0}>0$, such that

$$
e(\alpha, a)>3 D_{1}, \quad a \geq a_{0} .
$$

Note that $\alpha$ is bounded below in terms of $n$ and $D_{1}$.

We now divide the manifold $M$ into cones as follows: first divide the unit sphere in the tangent space at $p_{0}$ into $m$ closed domains $\left\{U_{1}, \ldots, U_{m}\right\}$ so that in each domain $U_{j}$, we have

$$
\angle(v, w) \leq \alpha, \quad \forall v, w \in U_{j} .
$$

Clearly $m=m\left(n, D_{1}\right)$ is bounded above in terms of $n$ and $D_{1}$. Define the cone $K_{j}$ by:

$x \in K_{j} \quad$ if and only if there is a minimal geodesic $\gamma$ from $p_{0}$ to $x$ with $\gamma^{\prime}(0) \in U_{j}$.

Now let $r \geq \max \left\{a_{0}, D_{1}\right\}$. On $\partial B_{p_{0}}(2 r)$, take a maximal set of points $\left\{p_{1}, \ldots, p_{k}\right\}$ such that $\operatorname{dist}\left(p_{i}, p_{j}\right) \geq r, i \neq j$. Then

$$
\begin{gathered}
\bigcup B_{p_{i}}(r) \supset \partial B_{p_{0}}(2 r) ; \\
B_{p_{i}}(r / 2) \cap B_{p_{j}}(r / 2)=\varnothing, \quad i \neq j .
\end{gathered}
$$

To estimate the bound on $k$, we divide the balls $B_{p_{i}}(r / 2)$ into $m$ families as follows: for each ball $B_{p_{i}}(r / 2)$, look at $\operatorname{Vol}\left(B_{p_{i}}(r / 2) \cap K_{j}\right), j=1, \ldots, m$. Fix a $j_{i}$ such that $\operatorname{Vol}\left(B_{p_{i}}(r / 2) \cap K_{j_{i}}\right)$ is maximal. Then

$$
\operatorname{Vol}\left(B_{p_{i}}(r / 2) \cap K_{j_{i}}\right) \geq \frac{1}{m} \operatorname{Vol}\left(B_{p_{i}}(r / 2)\right) .
$$

We denote

$$
B_{p_{i}}(r / 2) \cap K_{j_{i}}=B_{p_{i}}^{L, j_{i}},
$$

or simply $B_{p_{i}}^{L}$, and place the ball $B_{p_{i}}(r / 2)$ in the $j_{i}$ th family.

Now we estimate the number of balls in a cone $K_{j}$. Suppose $B_{p}^{L}$ has the smallest volume among all $B_{p_{i}}^{L, j}$ in this cone. Since $p$ may not be in $K_{j}$, we need the following

Lemma 4. Assume that $\operatorname{Ric} \mid B_{p}(R) \geq 0$. There exists a $\delta$ with $0<\delta<1$, depending only on $n$ and $m$, such that whenever a subset $W \subset B_{p}(R)$ has

$$
\operatorname{Vol}(W) \geq 1 / m \cdot \operatorname{Vol}\left(B_{p}(R)\right),
$$

then there exists $q \in W$ such that $\operatorname{dist}(q, p) \leq \delta R$. Hence $B_{q}((1-\delta) R) \subset$ $B_{p}(R)$.

Proof. If for any $q \in W$, $\operatorname{dist}(p, q)>\delta R$, then $W \subset A_{p}(R, \delta R)$. By Lemma 1 ,

$$
V(R, \delta R) / V_{p}(R) \leq \frac{R^{n}-\delta^{n} R^{n}}{R^{n}}=1-\delta^{n} .
$$

So $1-\delta^{n} \geq 1 / m$, i.e., $\delta \leq(1-1 / m)^{1 / n}$. Thus we can take

$$
\delta=(1-1 /(2 m))^{1 / n} .
$$

This finishes the proof of Lemma 4. 
Now we continue the proof of the theorem. By the above lemma and (4), there is a point $q \in B_{p}^{L}$ such that

$$
B_{q}((1-\delta) r / 2) \subset B_{p}(r / 2) \text {. }
$$

We construct a star-shaped set $U_{q}$ at $q$ as follows: $y \in U_{q}$ if and only if there is a point $x$ belonging to either $B_{q}((1-\delta) r / 2)$ or one of $B_{p_{i}}^{L, j}$ in the cone $K_{j}$ and there is a minimal geodesic $\gamma$ connecting $q$ and $x$ which passes $y$. Note that

(a) This geodesic $\gamma$ will not pass through $B_{p_{o}}\left(D_{1}\right)$ by the construction of the cone. To see this, note that if $x$ is in one of $B_{p_{i}}^{L, j}(r / 2)$, then both $x$ and $q$ are in $K_{j}$. The claim follows from (1) and the remarks following Lemma 3; if $x$ is in $B_{q}((1-\delta) r / 2)$ then the claim is immediate by the triangle inequality.

(b) The length of $\gamma$ is not bigger than $r / 2+2 r+2 r+r / 2=5 r$.

(c) $B_{q}((1-\delta) r / 2) \subset U_{q} \subset M-B_{p_{0}}\left(D_{1}\right)$.

Hence we can apply Lemma 2 . We have

$$
\operatorname{Vol}\left(U_{q}\right) / V_{q}((1-\delta) r / 2) \leq V^{0}(5 r) / V^{0}((1-\delta) r / 2)=10^{n} \cdot(1-\delta)^{-n}
$$

On the other hand let $N_{j}$ be the number of balls in the $j$ th family. By (4) and (6),

$$
\operatorname{Vol}\left(U_{q}\right) / V_{q}((1-\delta) r / 2) \geq \sum_{B_{p_{i}}^{L} \in j \text { th family }} \operatorname{Vol}\left(B_{p_{i}}^{L}\right) / V_{p}(r / 2) \geq N_{j} / m .
$$

So (7) and (8) imply $N_{j} \leq m \cdot 10^{n}(1-\delta)^{-n}$. Adding up the contributions from all $m$ families using (5), we have

$$
k \leq 10^{n} m^{2}\left[1-(1-1 /(2 m))^{1 / n}\right]^{-n} .
$$

Since $m$ depends only on $n$ and $D_{1}$ so does the right-hand side of (9). The theorem is proved.

Remark 2 . From the above proof, we see that we can replace $\partial B_{p_{0}}(2 r)$ by any subset $S$ satisfying $\sup _{x \in S} \operatorname{dist}\left(p_{0}, x\right) \leq C_{0} \operatorname{dist}\left(p_{0}, S\right)$; hence we have the following:

Suppose $\operatorname{Ric}_{M-B} \geq 0, K_{M} \geq H>-\infty$, and $B \subset B_{p_{0}}(D)$. Then $\forall \mu>$ $0, \forall C_{0}>0, \exists N>0$, and $r_{0}>0$ depending only on $n, \mu, H \cdot D^{2}$, and $C_{0}$, such that if $S$ is a subset satisfying

$$
\begin{gathered}
\sup _{x \in S} \operatorname{dist}\left(p_{0}, x\right) \leq C_{0} \operatorname{dist}\left(p_{0}, S\right), \\
r=\operatorname{dist}\left(p_{0}, S\right)>r_{0},
\end{gathered}
$$

we can find $p_{1}, \ldots, p_{k} \in S, k \leq N$, with

$$
\bigcup_{j=1}^{k} B_{p_{j}}(\mu \cdot r) \supset S \text {. }
$$

This more general form will be useful in the next section.

\section{Applications}

Corollary 1. Under the same assumption as in the theorem, the number of ends is finite and bounded by the same constant $N$ in the theorem. 
Proof. If not, take $r$ large enough so that in $M-B_{p_{o}}(r)$, there are $n>N$ separated ends. It is apparent that each such end $E$ requires at least one ball of radius $r$ to cover $E \cap \partial B_{p_{0}}(2 r)$. This contradicts the theorem.

There are several different definitions of the concept of "diameter growth." (Very roughly speaking, the diameter growth measures the intrinsic diameter of $\partial B_{p}(r)$ as a function of $r$.) As far as the question of linearity of diameter growth is concerned, they are all equivalent. See for example, [AG] and [S].

Corollary 2. Under the same assumption as in the theorem, the diameter growth of each end is at most linear.

Proof. Apply Remark 2 to $S$, a curve connecting two points on a distance sphere. We omit the details.

An immediate consequence of the theorem in the study of harmonic functions is the following:

Proposition 1. Assume that $\operatorname{Ric}_{M-B} \geq 0$, where $B \subset B_{p_{0}}(D)$ is a compact subset of $M$, and the sectional curvature of $M$ is bounded below. Let $E$ denote an end of $M$, that is, $E$ is an unbounded component of $M-B_{p_{0}}\left(r_{0}\right)$. Assume that $f(x)$ is a positive harmonic function defined on $E$. Then either $\lim _{x \rightarrow \infty} f(x)=\infty$ or $\lim _{x \rightarrow \infty} f(x)=a$ for some constant $a$, provided that one of the following conditions on $E$ holds:

(a) There is an $R_{0}>0$, such that $\pi_{1}\left(E-B_{p_{0}}\left(R_{0}\right)\right)=0$.

(b) $\forall r>0, \exists R>r$, such that the unbounded component $E-B_{p_{0}}(R)$ has a connected boundary.

(c) There is a sequence of connected (n-1)-dimensional compact submanifolds $S_{j} \subset E$, such that (i) $S_{j}$ and $S_{j+1}$ bound a connected compact region $D_{j}$ for $j=1,2,3, \ldots$; (ii) for some large $r, \cup D_{j} \supset E-B_{p}(r)$; (iii) $\operatorname{dist}\left(p_{0}, S_{j}\right) \rightarrow$ $\infty$; and (iv) for some constant $C$

$$
\max _{\boldsymbol{x} \in S_{j}} \operatorname{dist}\left(p_{0}, x\right) / \min _{x \in S_{j}} \operatorname{dist}\left(p_{0}, x\right) \leq C .
$$

Proof. We follow the same line of arguments as in the proof of Theorems 3.2 and 3.3 in [LT].

Since (a) implies (b) (see [AG, Proposition 4.3 and its proof]) which in turn implies (c), let us assume that the end $E$ satisfies condition (c). The proposition is a consequence of the following two lemmas.

Lemma 5 (Harnack inequality; see [Y, LY], also see [LT, Lemma 3.2]). There is a constant $C_{1}$, such that if $f(x)$ is a positive harmonic function on $E$ and $\operatorname{Ric}_{B_{q}(2 r)} \geq 0$, where $B_{q}(2 r) \subset E$, then

$$
|\nabla f(x)| / f(x) \leq C_{1} / r
$$

for any $x \in B_{q}(r)$.

Lemma 6. There is a constant $C_{2}>0$ independent of $j$ such that for any harmonic function $f(x)$ defined on $E$ and any large $j$, we have

$$
\max _{x \in S_{j}} f(x) \leq C_{2} \min _{x \in S_{j}} f(x) .
$$

Proof. Choose $y, z \in S_{j}$ with

$$
f(y)=\max _{x \in S_{j}} f, \quad f(z)=\min _{x \in S_{j}} f .
$$


Let $\gamma$ be a curve in $S_{j}$ connecting $y$ and $z$. Denote $r_{j}=\operatorname{dist}\left(p_{0}, S_{j}\right)$. Without loss of generality, we assume that $r_{j}>2 r_{0}$. Remark 2 implies that there are a bounded number of points $y=y_{1}, \ldots, y_{k}=z, k \leq C_{3}$, on the curve $\gamma$ such that

$$
\gamma \subset \bigcup_{i} B_{y_{i}}\left(1 / 4 \cdot r_{j}\right) .
$$

Now Harnack's inequality implies that

$$
f(y) / f(z) \leq C_{1}^{(2 k-1)} .
$$

This proves the lemma.

Now, Proposition 1 follows from the above lemma. The argument is as in the proof of Theorem 3.3 of [LT]. Namely, suppose that $f(x)$ is not bounded on $E$. We are going to show that

$$
\lim _{x \rightarrow \infty} f(x)=\infty .
$$

Since $f(x)$ is not bounded, there is a sequence of points $x_{j}, \lim \operatorname{dist}\left(x_{j}, p_{0}\right)=$ $\infty$, such that $\lim f\left(x_{j}\right)=\infty$. Suppose $x_{j} \in D_{i_{j}}$, then

$$
\max _{x \in D_{i_{j}}} f(x) \geq f\left(x_{j}\right) .
$$

By the maximum principle the maximum is either on $S_{i_{j}}$, in which case we denote $\sum_{j}=S_{i_{j}}$, or on $S_{i_{j}+1}$, in which case we denote $\sum_{j}=S_{i_{j}+1}$. We write $U_{j}$ for the domain bounded by $\sum_{j}$ and $\sum_{j+1}$. Then we have

$$
\min _{x \in \sum_{j}} f(x) \geq 1 / C_{2} \max _{x \in \sum_{j}} f(x) \geq 1 / C_{2} f\left(x_{j}\right) .
$$

Thus by the maximum principle $\min _{x \in U_{j}} f(x) \geq 1 / C_{2} \min \left\{f\left(x_{j}\right), f\left(x_{j+1}\right)\right\}$. Therefore $\lim _{x \rightarrow \infty} f(x)=\infty$.

Suppose that $f(x)$ is a bounded $E$. Let

$$
A=\lim _{r \rightarrow \infty}\left(\inf _{E-B_{p_{0}}(r)} f\right) ; \quad 0 \leq A<\infty .
$$

For any $\varepsilon>0$, there exists a sequence of points $x_{j}, \lim \operatorname{dist}\left(x_{j}, p_{0}\right)=\infty$, such that $A-\varepsilon<f\left(x_{j}\right)<A+\varepsilon$. Suppose $x_{j} \in D_{i_{j}}$. By the maximum principle $\min _{D_{i_{j}}} f$ is either on $S_{i_{j}}$, in which case we denote $\sum_{j}=S_{i_{j}}$, or on $S_{i_{j}+1}$, in which case we denote $\sum_{j}=S_{i_{j}+1}$. We write $U_{j}$ for the domain bounded by $\sum_{j}$ and $\sum_{j+1}$. Also, there is a $j_{0}$, such that

$$
\bigcup_{\bigcup_{i>j}} U_{i} f>A-\varepsilon
$$

for any $j \geq j_{0}$.

Let $g=f-(A-\varepsilon)$, which is a positive harmonic function on $\bigcup_{i>j_{0}} U_{i}$. Lemma 6 implies that for sufficiently large $j$,

$$
\max _{x \in \sum_{j}} g(x) \leq C_{2} \min _{x \in \sum_{j}} g(x) .
$$

Hence

$$
\max _{x \in \sum_{j}} g(x) \leq C_{2}\left\{\min _{x \in \sum_{j}} f-(A-\varepsilon)\right\} \leq 2 C_{2} \varepsilon
$$




$$
\max _{x \in \sum_{j}} f \leq\left(2 C_{2}-1\right) \varepsilon+A
$$

Since $\varepsilon$ is arbitrary, by the maximum principle again we have

$$
\lim _{j \rightarrow \infty}\left(\sup _{\bigcup_{i>j} U_{i}} f\right) \leq A \text {. }
$$

This shows that $\lim _{x \rightarrow \infty} f=A$. The proposition is proved.

We intend to further investigate the structure of the space of positive harmonic functions elsewhere.

\section{ACKNOWLEDGMENT}

I would like to thank Professor J. Cheeger for introducing me to this problem and for inspiring conversations. I would also like to thank Professor D. Gromoll for several helpful discussions.

Added in proof. The original version of this paper was submitted for publication in May 1990.

At the AMS Summer Institute on Differential Geometry held at U.C.L.A., July $1990, \mathrm{P}$. Li announced that he and L. Tam had proved that $\mathrm{Ric}_{M-B} \leq 0$ implies that $M$ has finitely many ends (without assuming a lower bound on the sectional curvature), and the number of ends depends only on $n$ and $H \phi^{2}$, where $\phi$ is the diameter of $B$ and $\operatorname{Ric}_{B} \geq(n-1) H$. Their approach is analytic in nature. At about the same time, $M$. Cai independently proved this same result by purely geometric means.

Cai's argument suggested to us that our original approach could be slightly modified by using the triangle inequality in place of Toponogov's Theorem, thus eliminating the requirement of an arbitrary lower bound on the sectional curvature; i.e. we can establish the ball covering property assuming only $\operatorname{Ric}_{M-B} \geq 0$, and the covering number depends only on $n$ and $H D^{2}$, where $H$ is as above and $D$ as in the theorem. (Ball covering is not treated in either of the aforementioned works.)

In fact, it is only necessary to modify the definitions of the subsets $U_{j}$ and of the corresponding cones $K_{j}$. We can define $\left\{U_{1}, \ldots, U_{m}\right\}$ as subsets of $\partial B_{p_{0}}\left(2 D_{1}\right)$ such that $d_{M}\left(U_{j}\right) \leq 2 D_{1}$, while $K_{j}$ is the star-shaped set consisting of all minimal geodesics emanating from $p_{0}$ that intersect $U_{j}$. Then the triangle inequality easily implies that any minimal geodesic connecting two points in $K_{j}$ which are sufficiently far away from $p_{0}$ will not intersect $B$. The rest of the construction remains unchanged. Details will be given elsewhere.

\section{REFERENCES}

[AG] U. Abresch and D. Gromoll, On complete manifolds with nonnegative Ricci curvature, J. Amer. Math. Soc. 3 (1990), 355-374.

[BC] R. Bishop and R. Crittenden, Geometry of manifolds, Academic Press, New York, 1964.

[CGT] J. Cheeger, M. Gromov, and M. Taylor, Finite propagation speed, kernel estimates for functions of the Laplace operator, and the geometry of complete Riemannian manifolds, $\mathrm{J}$. Differential Geometry 17 (1982), 15-53.

[GLP] M. Gromov, J. Lafontaine, and P. Pansu, Structures métriques pour les variétés Riemanniennes, Nathan, Paris, 1982. 
[LT] P. Li and L. Tam, Positive harmonic functions on complete manifolds with non-negative curvature outside a compact set, Ann. of Math. 125 (1987), 171-207.

[LY] P. Li and S. T. Yau, On the parabolic kernel of the Schrödinger operator, Acta Math. 156 (1986), 153-201.

[S] Z. Shen, Finiteness and vanishing theorems for complete open Riemannian manifolds, Bull. Amer. Math. Soc. 21 (1989), 241-244.

[Y] S. T. Yau, Harmonic functions on complete Riemannian manifolds, Comm. Pure Appl. Math. 28 (1975), 201-228.

Department of Mathematics, State University of New York at Stony Brook, Stony BROOK, NEW YORK 11794 